\title{
Saúde ur bana: "a cidade é uma estranha senhora, que hoje sorri e amanhã te devora"**
}

\author{
Urban health: "the city is a strange lady, \\ smiling today, devouring you tomorrow"
}

Waleska Teixeira Caiaffa ${ }^{1,2}$

Fabiane Ribeiro Ferreira ${ }^{1}$

AlineDayrell Ferreira ${ }^{1,3}$

Claúdia Di Lorenzo Oliveira ${ }^{1,4}$

Vitor Passos Camargos ${ }^{1}$

Fernando Augusto Proietti ${ }^{1,2}$

*Luís Enriquez, Sérgio Bardotti eChico Buarque de Hollanda. A cidadeideal, música do disco Os saltimbancos.

${ }^{1}$ Observatório deSaúde Urbana, Faculdadede Medicina, UFM G. Av. Alfredo Balena 190/625, Santa Efigênia. 30130-100 Belo H orizonte M G. wcaiaffa@medicina.ufmg.br ${ }^{2}$ Departamento de M edicina Preventiva e Social, FaculdadedeM edicina, UFM G.

${ }^{3}$ Departamento $M$ aternoInfantil eSaúde Coletiva, Escola de Enfermagem, UFMG.

${ }^{4}$ Fundação H emominas, Belo H orizonte, M G.
Abstract More than half of the world's population is living in cities and the world is turning more and more urbanized. This literature review explores the ramifications of urban transformation, showing how cities take shape and impact human health in our times. While cities can offer positive opportunities, their negative impacts re lated to the lack of social organization, precarious urban living and working conditions, lack of governance and opportunities as well as the lack of strategies for promoting social equity tend to increase the adverse effects on the health of the urban communities. The article also advocates the study of urban health as a branch of knowledge related to public health, proposing a common conceptual model and taxonomy for urban health. Future directions for research and practice are discussed in the light of some examples from the Brazilian scientific literature.

Key words U rban health, U rbanization, Inequalities, Cities
Resumo M ais da metade dos habitantes da terra está vivendo nas cidades, e o mundo está se tornando mais emais urbano. Esteartigo considera, a partir de revisão bibliográfica, que 0 ambiente urbano influencia a saúde e os comportamentos humanos, apontando para a necessidade do meIhor entendimento dos determinantes da saúde das populações vivendo nas cidades. Explora as transformações urbanas oferecendo uma visão sobre a conformação das ci dades contemporâneas e os impactos sobre a saúde humana. Sugere que, enquanto as cidades podem representar oportunidades positivas, impactos desiguais negativos relacionados à falta de organização social, precárias condições urbanas e de trabalho, falta de governança, de oportunidades, e de capacidade de desenvolver políticas para ações tendem a ampliar os efeitos adversos sobre a saúde das pessoas. Discutea saúde urbana como um ramo de conhe cimento aninhado na saúde pública, possi bilitando re-pensar avaliações deimpacto à saúde a partir de intervenções não necessariamente originadas da saúde. Propõe arcabouço de um modelo conceitual, além de uma taxonomia comum para a saúde urbana. A presenta direções futuras de investigação e prática, contemplando especificidades analíticas, sob a luz de alguns exemplos da literatura científica brasileira.

Palavras-chave Saúde urbana, U rbanização, Iniqüidades, Cidades 
Introdução

As cidades e seus residentes

As cidades e o viver nas cidades representam uma realidade mundial. Em 1800, apenas duas cidades, Londres e Pequim, tinham mais de um milhão de habitantes. Cem anos depois, eram quase dez com este porte e este número triplica em 1950. Em 1980, duzentos anos depois, são quatrocentas as cidades com este perfil, espal hadas por todo o mundo $0^{1,2}$.

Até muito recentemente, a população mundial vivia em áreas rurais. Em 2008, estima-se que, pela primeira vez na história da humanidade, a metade da população do mundo, 3,3 biIhões de indivíduos, estará vivendo nas cidades. Em 2030, aproximadamente 5,0 bilhões de pessoas, ou seja, dois terços da população mundial residirão em áreas urbanas. No mesmo período, a população rural deverá diminuir para cerca de 28 milhões de habitantes. Globalmente, portanto, todo o crescimento populacional futuro está previsto ocorrer nas cidades ${ }^{3}$.

São marcantes as alterações no eixo evolutivo da construção das cidades e suas respectivas organizações socioespaciais4. Do ponto "zero", historicamente representado pelos primeiros agrupamentos humanos, surge a "cidade política", caracterizada pela vida organizada com taxas e tributos. Esta sofre inflexão do agrário para o urbano, sendo substituída pela "cidade comercial". As transformações na produção, distribuição, troca, consumo e nas relações sociais originam um complexo movimento humano num processo de implosão e explosão ocasionado pelo êxodo rural, caracterizando a transição da "cidade comercial" para a "cidade industrial". Tal mudança associa-se à consolidação do modo capitalista de produção. $\mathrm{Na}$ "cidade industrial", observa-se a extensão e esgarçamento do tecido urbano, com subordinação completa da não cidade 5 .

M undialmente, a distribuição das pessoas vivendo nas cidades e o crescimento populacional não são homogêneos. $M$ ais de dois terços da população concentram-se na África, Ásia eAmé rica Latina ${ }^{2}$. N estas regiões, enquanto um quinto do crescimento ocorrerá nas grandes cidades, estima-se que quase metade será naquelas de menor porte, ondea maioria da população mundial estará vivendo no século $21^{6}$.

No Brasil, o fenômeno das cidades não é diferente. A proporção da população residindo em áreas urbanas passou de 31,3\% em 1940 para $81,2 \%$ em 2000. Este crescimento foi maior nas décadas de 1950 e 1960, mas somente nos anos setenta, observou-se a grande inflexão do urbano sobre o rural, que se tornou cada vez mais pronunciada, com o passar das décadas?

A expansão urbana também não tem sido homogênea no que se refere à região geográfica. O corre de forma mais acentuada nas regiões metropolitanas do sudeste, acirrando os desequilíbrios econômicos esociais, tanto entre cidades quanto dentro das cidades. Também não são homogêneas as taxas de crescimento das cidades, sendo detectadas maiores taxas naquelas de porte médio. Entre 1991 a 2006, cidades com 100 a 500 mil habitantes tiveram crescimento de $41 \%$, comparado ao crescimento médio geral de $27 \% 8$.

Agravado pelo declínio da taxa defecundidade e conseqüente decréscimo na velocidade do crescimento populacional após a década de 1960 , a mudança do perfil das cidades brasileiras culmina com o auge do ciclo de expansão das migrações, com intenso fluxo rural-urbano, responsável por $53 \%$ do crescimento da população urbana ${ }^{7}$. Substanciais mudanças no âmbito da mortalidade se adicionam e conformam os componentes principais da chamada transição demográfica do país9.

Neste contexto, a "urbanização extensiva" constitui condição fundamental para a compreensão do espaço social contemporâneo brasileiro ecaracteriza-se pela materialização do processo de produção e reprodução, aspecto central da urbanização e da "cidade industrial", e se estende para além do aglomerado urbano, ocupando todo o espaço social ${ }^{10}$.

\section{O crescimento desigual das cidades}

Quanto ao perfil de crescimento das cidades no mundo, este varia de acordo com o desenvolvimento regional. Enquanto em regiões consideradas mais desenvolvidas estese dá de forma mais gradual, nas regiões menos desenvolvidas o crescimento se dá de forma vertiginosa, com previsão da suplantação do crescimento urbano sobre o rural em $2020^{3}$.

Tais informações coincidem com estimativas mundiais estratificadas pelo Índice de D esenvolvimento Humano (IDH). Regiões com baixos IDH $(<0,50)$ terão um crescimento populacional médio anual de 3,1 quando comparado com 1,1 das regiões de médio IDH $(0,50-0,79)$ e 0,4 de alto IDH $(\geq 0,80)^{10}$. Considerando a velocidade destecrescimento pelos mesmos estratos deIDH, observam-se maiores inflexões em áreas de baixo IDH, estimado em $11,7 \%$, quando compara- 
do a 4,7\% das áreas com altos IDH ${ }^{11}$. Como conseqüência do crescimento populacional e da urbanização desigual, estima-se que, ao atual um bilhão de pessoas vivendo em áreas consideradas vulneráveis, somar-se-á mais um bilhão em trinta anos, e outro bilhão até 2050, se as condições assim se mantiverem ${ }^{12,13}$.

No Brasil, ambos os processos (crescimento populacional e urbanização desigual) ocorreram principalmente devido à "periferização", após a década de 1970, com impacto direto e indireto nas condições demoradia esaúde da população ${ }^{14}$.

\section{0 viver nas cidades}

Viver na cidade é certamente a mudança de mográfica mais importante ocorrida nas últimas décadas, neste e no século passado. Claude de Saint Simon, no século $X I X^{15}$, foi o pioneiro a vincular a idéia de cidade com a metáfora dos organismos vivos gigantescos, sem precedentes na história humana, permeado por redes que os alimentam e mantêm seu funcionamento.

Assim, cidades contemporâneas representam metabolismos complexos que, com suas redes e conexões, seestendem globalmente, com relevantes implicações em saúde, incluindo a agudização dos problemas sociais como a violência, os acidentes de trânsito, a presença de doen ças emergentes ere-emergentes, transmissíveis ou não ${ }^{16,17}$.

No Brasil, o impacto do surgimento das cidades contemporâneas nos últimos cinqüenta anos, tal como ocorreu anteriormente na Europa, interligou-se à profunda mudança do perfil demográfico do país, com declínio do coeficiente de mortalidade geral, redução da mortalidade infantil, aumento da expectativa de vida e conseqüente modificação do perfil epidemiológico ${ }^{9}$. Este processo, o qual $0 \mathrm{mram}^{18}$ descreveu como de transição epidemiológica, caracteriza-se pela evolução progressiva deum perfil de alta mortalidade por doenças infecciosas para um outro ondepredominam os óbitos por doenças cardiovasculares, neoplasias, causas externas e doenças consideradas crônico-degenerativas.

Assim, de forma cosmopolita, o viver na cidade pode ser benéfico, conhecido como a "vantagem do urbano", ou pode ser nocivo, conhecido como a "penalidade do urbano"19. Independente das vantagens e desvantagens, a cidadecontemporânea reforça a idéia de que seus moradores estão constantemente sen do afetados por sua dinâmica. Nesta direção, o conceito de saúde deveria incorporar o cotidiano dos indivíduos vi- vendo nas cidades, sob a ótica ampliada de que o estudo individualizado dos fatores determinantes na saúdee suas conseqüências, antes reducionista, não pode ignorar as relações de interdependência que existem entreo indivíduo eo meio físico, social e político onde ele vive e se insere. Surge, em decorrência, a discussão sobre a saúde urbana ${ }^{4,19-21 .}$

\section{D efinição do objeto}

\section{Saúde urbana: antecedentes}

Segundo recente consenso da Organização Pan-Americana da Saúde²2, foi em 1968 o primeiro registro correlacionando a mortalidade urbana e condições de saúde ${ }^{23}$. Somenteem 1993, a Organização M undial deSaúde(OM S) despertou para a crise na saúde urbana, entendo-a então como um desafio, resultando em encontro de pesquisadores da área em Kobe, Japão, em 199624. Ainda nesta década iniciaram-se os movimentos das cidades saudáveis que configuraram uma rede entre 2000 e 2006. N este mesmo período, foi fundada a Sociedade Internacional de Saúde Urbana, que muito tem contribuído para o dinamismo da área, inclusive com a publicação periódica do J ournal of U rban $\mathrm{H}$ ealth da Academia de M edicina de N ova Iorque ${ }^{25}$.

No Brasil, por iniciativa dos Cadernos deSaúde Pública e coincidente com o lema central "Um Olhar sobre a Cidade" do IV Congresso Brasileiro deEpidemiologia (EPI 2004) realizado em $\mathrm{Re}$ cife, Pernambuco, em 200526, foi publicado o fórum de saúde urbana ${ }^{10,20,27,28}$. Em 2007, com 0 recente relatório da Rede de Conhecimentos dos Entornos Urbanos, conhecida como KNUS (KnowledgeN etwork on U rban Settings), produzido por solicitação da Comissão dos D eterminantes Sociais em Saúde da Organização M undial de Saúde (CDSS - OMS), a saúde urbana ficou em evidência, marcando claramente a transcendência dos efeitos da urbanização sobre a saúde humana29,30.

Paulatinamente, instituições signatárias das $N$ ações Unidas, de ciência e de iniciativas privadas, tais como a Fundação Rockefeller ${ }^{31}$, vão convergindo para a discussão do tema. Destaque especial merece a iniciativa da O rganização PanAmericana deSaúde com a realização do Primeiro Fórum Regional de Saúde Urbana, para uma discussão do marco conceitual e desenvolvimento de uma ação nas Américas, em 200722. 


\section{Conceitos básicos em saúde urbana}

Antes de tratarmos propriamente do objeto saúde urbana, alguns conceitos emergem. M ais que contribuir para a própria definição do objeto, tais conceitos podem auxiliar no entendimento das especifici dades analíticas e práticas da definição de saúde urbana. São eles: urbano, urbanicidade e urbanização ${ }^{32}$.

O termo urbano veio do latim e tem dupla conotação: de urbanum ( $\operatorname{arado}$ ) e de povoação. Possivelmente representa a forma física do espaço delimitado pelo sulco do arado dos bois sagrados que marcava o território da produção e vida dos romanos. Daí, surgem os termos urbe e urbs, este último referindo-se à cidade de Roma, também conhecida como cidade-império. Posteriormente, o termo urbano foi resgatado no sé culo XVI para sereferir à cidade-sededo I mpério Britânico. Surge também a palavra city, relacionada apenas ao centro financeiro ${ }^{10}$. Assim, a definição de urbano, desde sua origem, se faz naturalmente dentro da estreita inter-relação com seu oposto, o rural, tornando o termo, desde sua concepção, polêmico e dinâmico.

Mas, como caracterizar o território urbano? Embora exista uma tendência em direção a um consenso sobreas definições básicas deste conceito, há ainda o desafio de validação no campo da literatura e da prática. Wirth, em $1938^{33}$, propôs caracterizar a área urbana de acordo com o tamanho, a densidade e a heterogeneidade populacional. Entretanto, sendo uma definição funcional ou administrativa, cada país ou região tem adotado sua própria tipologia4. Segundo o relatório da OPAS ${ }^{22}$, dos 228 países para os quais as Nações Unidas têm informações, quase metade usou a definição administrativa de "áreas urbanas" - "as pessoas que vivem na capital do país"; $22 \%$ utilizaram o critério do tamanho e densidade; $17 \%$ levaram em conta as características funcionaistais como atividade econômica e $11 \%$ não apresentaram uma definição para este termo. Além disso, alguns países usaram o termo para definir toda a população do paísou qual quer parte dele $e^{22}$. Vale ressaltar que estas definições também variam deforma significativa, não somente entre países, mas ao longo do tempo, introduzindo possivelmente viés de classificação nas comparações entre e mesmo dentro de um país s,32 $^{4}$.

No Brasil, o Instituto Brasileiro de Geografia e Estatística (IBGE) define cidade como a localidade com o mesmo nome do município a que pertence ( sede municipal) e onde está sediada a respectiva prefeitura, excluídososmunicípiosdas capitais. Portanto, todas as localidades com estas características, onde habitam 5.000 pessoas ou mais, seria considerada uma cidade. Já área urbana éo espaço geográfico interno ao perímetro urbano de uma cidade ou vila, definido por lei municipal, sendo área rural aquela externa ao perímetro urbano ${ }^{34}$.

Aspectos contemporâneos como intensidade, ritmo, duração e resposta a mudanças, assim como definiçõesadministrativas eatividadeseconômicas são pouco ou nada contempladas nas definições acima, ficando, portanto, ainda pendente uma conceituação mais abrangente. Didaticamente, compilando publicações atuais na área ea mais recente revisão produzida pela OPAS, de forma abrangente e simples, propõe-se o termo "urbano" para referir as cidades e seu entorno, o termo "urbanicidade" para o estudo de condições de saúde relevantes a áreas urbanas num momento (visão transversal) e "urbanização" como o complexo processo mediante o qual a cidadecresce (ou diminui), modifica einfluencia a saúde (visão longitudinal)19,22,32,35.

Outros três termos são aqui adicionados, considerando seu grande uso atual, coincidindo com o grande movimento de investigação dos determinantes sociais em saú de $e^{30}$ e por se revelarem úteis no processo de entendimento conceitual da saúde urbana. São el es: desigual dade, definido como as diferenças sistemáticas entre grupos populacionais em termos de indicadores de saúde ${ }^{30,35,36}$, iniqüidade, definida como as desigualdades injustas e evitáveis $s^{30,36}$ e, finalmente, vulnerabilidade, definida como a capacidade diferenciada deindivíduos, grupos, classes deindivíduos ou mesmo regiões ou lugares de manejar riscos, baseados em suas posições, nos mundos físicos e social ${ }^{37,38}$.

\section{Como conceber saúde urbana?}

Em direção ao conceito desaúdeurbana, três pontos podem ser sistematizados: (1) que a urbanização, antes esperada produzir somente efeitos benéficos, conhecida como as "vantagens urbanas", pode acarretar danos sociais, econômicos e ambientais de grande impacto, difíceis de mensurar completamentenos dias atuais; (2) que os atributosfísicos e sociais (contexto) da cidade e seus bairros e/ou vizinhanças podem afetar a saúde dos indivíduos; (3) que a ocorrência dos eventos relacionados à saúde, numa visão mais abrangente, estaria associada a atributos dos indivíduos aninhados no "lugar urbano" assim como o somatório das propriedades do agrega- 
do destes indivíduos (composição), indo mais além de seus atributos puramente individuai $s^{22}$.

Incorporando as propriedades do lugar, aqui entendido como a cidade eseu entorno, eo papel do ambientefísico e social (o contexto) em moldar a saúde das pessoas, parece plausível entender a cidade/urbano como exposição, modulando de forma benéfica ou danosa a saúde de suas populações residentes ${ }^{39}$. Como conseqüência, a incorporação de variáveis de contexto que capturem as características do "lugar urbano" onde os indivíduos vivem é mandatária nesta nova visão do processo saúde-doenç ç22,39. Ademais, intervenções não necessariamente originadas da saúde repercutem nos eventos em saúde, possibilitando re-pensar novas avaliações de impacto à saúde ${ }^{40}$. E assim, a saúde urbana pode ser considerada como um ramo da saúde pública que estuda os fatores de riscos das cidades, seus efeitos sobrea saúde e as relações sociais urbanas ${ }^{22,39}$. Os atuais pilares da saúde urbana são: 0 adensamento de populações; o papel do ambiente físico e social como modelador da saúde das pessoas; a necessidade de aferir os fenômenos tendo como objeto as desigualdades injustas e evitáveis do ambientefísico, social eem saúde ea governança ou governabilidade, como propostas para as soluções para as iniqüidades ${ }^{22}$.

\section{Arcabouço do model o conceitual de saúde urbana}

Propõe-se, portanto, modelo conceitual de saúde urbana, cuja característica é uma rede interligada de determinantes, tendo como cerne fundamental a proposta de que o social e o físico definem o contexto urbano esão modulados por fatores (proximais e distais) e atores em níveis múltiplos. Tendências globais, governos nacionais e locais, sociedade civil, mercado e o setor privado modulam o conjunto nos quais estes fatores locais operam ${ }^{39,41}$.

0 referencial teórico espel ha-se no sugerido por Galea \& Vlahov ${ }^{42}$, recentemente adaptado para o Relatório daRede deEspecialistas em M eios ou Assuntos Urbanos (KNUS) para a Comissão dos Determinantes Sociais da OM S29. 0 modelo adaptado na Figura $1^{29,42-44}$ ilustra como a saúde da população urbana representa uma função das influências mundiais, nacionais e das caracterís-

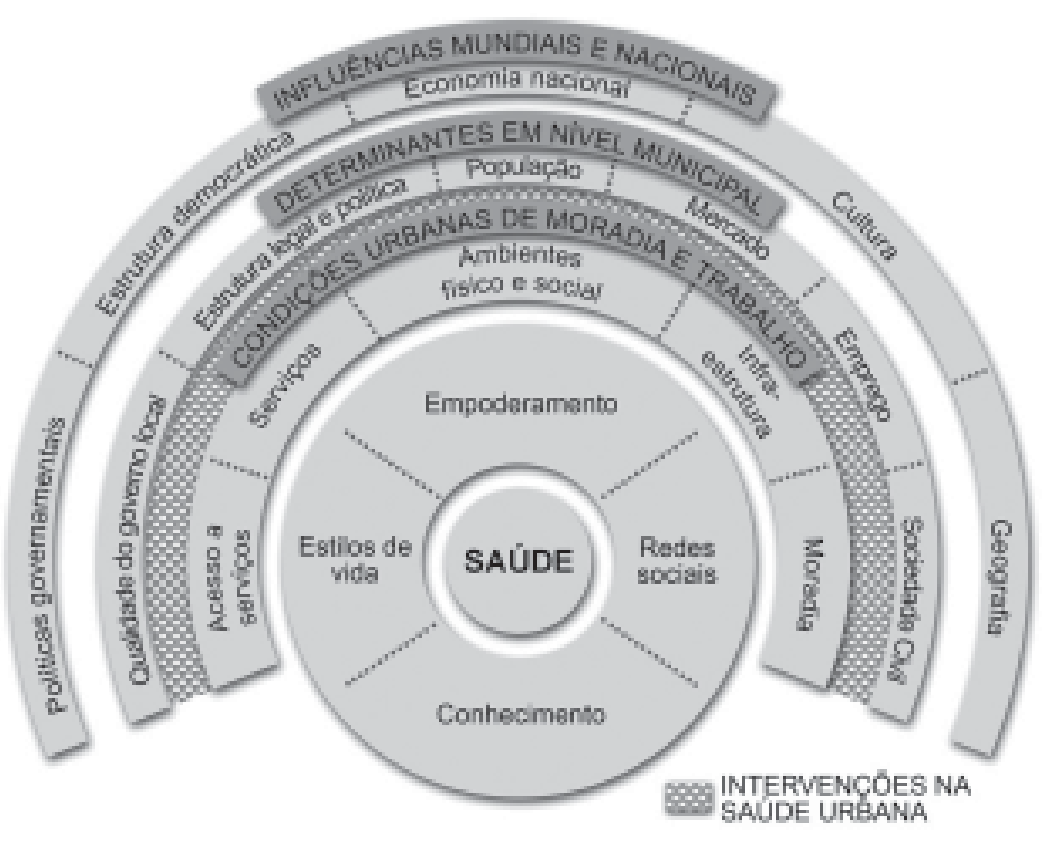

Fonte: Adaptado deWorld Health Organization ${ }^{29}$, Galea \& Vlahov ${ }^{42}$, Freudenberg $\mathrm{N}$ et al.$^{43}$ eKrieger $\mathrm{N}^{44}$.

Figura 1. M odelo conceitual para Saúde U rbana. 
ticas do município. Destaca como fatores proximais as condições urbanas de moradia e trabaIho, considerados fatores primariamente modificáveis. Sugerequeintervenções mais promissoras para modificar a saúde urbana são aquelas que procuram fazer mudanças específicas nessas condições de vida, e ainda assume 0 ambiente urbano num contexto amplo incluindo os ambientes físicos, sociais, econômicos e políticos.

0 entendimento é de que a forma como cada componente do modelo influencia o resultado do processo saúde-doença não élinear. Ou seja, cada um dos fatores interage entre si, mas também podeter um efeito independenteno resultado do processo. Variáveis mais proximais como as que compõem o nível "condições urbanas de moradia e trabal ho" caracterizam o cotidiano das pessoas que moram em áreas urbanas e podem ser vistas como condições preexistentes que a saúde pública procura modificar, intermediar, ou ainda, advogar, como o caminho pelo qual as intervenções podem levar à melhoria das condições de saúde.

O resultado, ter ou não um estado saudável no ambiente urbano, representa a finalidade da atenção da saúde pública que, nestemodelo, pode ser abordado numa perspectiva mais focalizada ou mais abrangente. Ou seja, permite análise em um único nível, independente se distal ou proximal, e em níveis múltiplos, remetendo, naturalmente, a questões metodológicas quepressupõem uma discussão norteadora para a aferição, comparação e mensuração da efetividade das estratégias de intervenção.

\section{Métodos}

Saúde urbana e as questões metodológicas

O estudo da saúde urbana envolve diversas e múltiplas disciplinas, com seus respectivos campos emétodos específicos. M étodos epidemiológicos têm sido empregados, ocasionalmente em concerto com outras disciplinas, no sentido de iluminar questões na investigação da saúde urbana, guiando, potencialmente, intervenções ${ }^{42}$.

Considerando o arcabouço teórico acima discutido, que inclui vários construtos e inúmeras variáveis latentes, questões metodológicas seavolumam no sentido da construção de modelos epidemiológicos deaferição, incluindo base amostral, definição deindicadores einstrumental analítico. São complexas e vêm sendo amplamente discutidas no campo da saúde urbana $a^{42,43}$.
População de estudo, base amostral e indicadores

A conformação da base populacional e simultaneamente amostral se remete à definição do conjunto de indivíduos vivendo e, portanto, expostos a um ambiente urbano demarcado geográfica ou socialmente. Refere-se, portanto, à população adstrita a uma determinada área em estudo. Assim, características como o tamanho, densidade, heterogeneidade populacional, intensidade, ritmos, duração e capacidade de resposta às mudanças e/ou intervenções podem ser levadas em consideração. Quando restrito às comunidades, prevalece simultaneamente ou não, 0 critério geográfico e de identidade social. 0 primeiro inclui unidades geográficas como municípios, bairros, áreas postais, quarteirões, setor censitário. 0 segundo é caracterizado pela vizinhança, comunidades, vilas, laços sociais e laços comunitários ${ }^{43}$.

Quanto aos indicadores, didaticamente, de acordo com o modelo proposto (Figura 1), podem ser compreendidos como aqueles referentes às condições de saúde ou sua ausência, de conhecimento, de redes sociais, deempoderamento, de estilo de vida, de acessos a bens e serviços, do ambiente ou contexto, de fatores econômicos e demográficos e de governabilidade. Envolvem construtos diversos com inúmeras variáveis ou classes latentes, que não podem ser medidas diretamente, tais como qualidade de vida, capital social, satisfação, felicidade, bem-estar ${ }^{45}$. Todos eles constituem temas complexos e em construção no campo da saúde $e^{46-48}$.

Instrumental analítico

Não há soluções simples para os problemas multidimensionais da saúde no contexto das cidades. Várias estratégias epidemiológicas podem ser adotadas, com o objetivo de identificar fatores associados às características do ambiente (lugar) na modulação da saúde dos indivíduos residentes deste lugar. Destacam-se a estratégia ecológica, a contextual, a hierárquica ea observação sistemática do ambiente $42,43,46,47,49,50$.

As análises ecológicas consideram associações entre fatores em nível de grupo ou agregados. Têm o propósito de gerar hipóteses sobre as características das cidades que podem afetar a saúde, permitindo comparações.

Ao contrário das análises ecológicas queapresentam capaci dade limitada de determinar como as características afetam a saúde dos indivíduos, 
as análises contextuais consideram o urbano como variável com efeito fixo nosindivíduos, isto é, o urbano tem o mesmo efeito em todos os indivíduos da análise, permitindo utilizar desde as tabelas de contingência até análi ses de regressão. Permitem estimar a chance de um grupo de indivíduos apresentar ou não um determinado evento em saúde quando expostos a contextos urbanos e não urbanos, por exemplo ${ }^{42}$.

Embora relativamente comuns na investigação em saúde urbana, as inferências que podem ser extraídas da análise contextual sobre como 0 modo de vida urbano pode afetar a saúde apresentam limitações. Esta análise assume que uma característica urbana em uma dada cidade éigual para todos os indivíduos, obscurecendo o fato de que al guns terão acesso diferenciado a determinadas características ou equipamentos urbanos. Acrescenta-se também o recente conhecimento de que os impactos ambientais também se têm revelado com diferenciais em função da capacidade distinta dos grupos sociais se afastarem das fontes de risco como revelados por pesquisas empíricas socioespaciais sobre desigualdades ambientai $s^{38}$. Assim, fornecendo resultados globais do dano à saúde em ambientes urbanos específicos, a análise contextual tem utilidadelimitada na contribuição do entendimento etiológico dos eventos relacionados à saúde.

Recentemente utilizadas na saúde urbana, as análises de multinível permitem compreender "como" as características das cidades ou de unidades dentro de uma cidade contribuem para a saúde dos indivíduos nela aninhados. Esta estratégia reconhecea importância de se estudar interações em múltiplos níveis e estão presentes em vários trabalhos na literatura. São ferramentas úteis no estudo da saúdeurbana epermitem considerar se características do urbano explicam a variabilidade entre indivíduos no que diz respeito à saúde, após controlar por características individuais relevantes. Permite considerar o quanto as características dos múltiplos níveis do viver urbano podem estar associadas à saúde, bem como entender o papel das redes sociais inseridas nesses níveis de escalas geográficas $5^{42,46,47}$.

Diversos estudos têm abordado o entorno do individuo, seja para mensurar a sua influência no hábito de vida, seja para compreender como as relações de vizinhança podem afetar a saúde das pessoas. M edir as propriedades social efísica em uma vizinhança constitui hoje um dos maiores desafios conceitual, metodológico e operacional para a incorporação destetipo de informação em estudos em saúde urbana. U ma op- ção é a inclusão da percepção do indivíduo acerca de sua vizinhança, que tem como vantagem a informação peculiar de aspectos da organização e estrutura do bairro/vizinhança, e como desvantagem o fato de que sua percepção estará sempre permeada por valores, experiências e papel social dentro de uma dada comunidade, introduzindo importante fonte de bias.

Uma alternativa contemporânea tem sido a inclusão de medidas resultantes de observação direta das condições físicas e interações sociais de um bairro/vizinhança (O bservação Social Sistemática). Essa abordagem, ainda pouco freqüente no Brasil, permite a obtenção de informações e medidas que independem da percepção individual eque eventualmentea complementam. Esta metodologia vem sendo muito utilizada em estudos que objetivam medir a relação entre características da vizinhança e atividade física. Algumas vantagens do método, como a cobertura de maiores áreas e a observação do comportamento e do ambiente num mesmo momento, são citadas por Suminski et al. ${ }^{49}$, têm como referencial teórico a ecometria discutida por Raundenbush \& Sampson ${ }^{50}$ e foram recentemente revisadas por Proeitti et al. ${ }^{51}$.

M odel os de estudos epidemiológicos em saúde urbana

Independente do método analítico adotado, estudos epidemiológicos que preenchem 0 arcabouço teórico da saúde urbana podem ser didaticamente divididos, de acordo com grupos de comparação, em: peculiarmente urbanos, urbano-rural, interurbano (entre cidades) e intraurbanos (entre vizinhanças).

Model os tipicamente urbanos nos remetem a estudos em que a exposição é peculiar ao ambienteurbano. D estacam-se exposições como poluição e transporte nas grandes cidades, ruídos no metrô e pontos de ônibus ${ }^{52,53}$. Estes estudos geralmente são ecológicos ou transversais e, naturalmente, apresentam limitações próprias. Como exemplo, e focalizando nas desvantagens do viver no ambiente urbano, podemos citar o recente estudo ecológico realizado na cidade de São Paulo, SP, no qual foram observadas significativas correlações das hospitalizações por causas respiratórias e cardiovasculares associadas à contaminação atmosférica ${ }^{54}$.

O segundo tipo de estudo objetivou comparar como o urbano em relação ao rural afeta a saúde das pessoas. Neste exemplo, um estudo transversal utilizando análise contextual demons- 
trou quea realização de exames preventivos como a mamografia e o exame de Papanicolau em mulheres urbanas com 25 anos ou mais foi cerca de duas vezes mais freqüente que em mulheres da área rural da mesma idade ${ }^{55}$. N este exemplo, podemos comprovar os efeitos benéficos na saúde do viver nas cidades, embora não nos permita conhecer 0 acesso aos serviços, fornecendo somente resultados globais do ganho em saúde no contexto urbano, com limitada contribuição para o entendimento etiológico deste processo.

0 terceiro tipo de estudo epidemiológico permite investigar como as cidades podem afetar a saúde das pessoas através da comparação interurbana, isto é, entre cidades, dentro de um país ou entre países. Utiliza a cidade como unidade de análise e compara diferentes cidades de forma a obter conclusões acerca das características associadas à saúde. Tais estudos, embora com características de vigilância, podem gerar hipóteses etiológicas para explicar o "porquê" das diferenças na saúde entre áreas urbanas. Se correlacionarmos a proporção da população vivendo em áreas urbanas de municípios brasileiros com mais de 30 mil habitantes e a taxa média de óbitos anual por agressão, no período de 2000 a 2005, encontraremos uma clara tendência positiva ( $\mathrm{Fi}$ gura 2). Estes estudos contribuem com a habilidade de discernir características das cidades que podem provocar dano à saúde das pessoas e su-

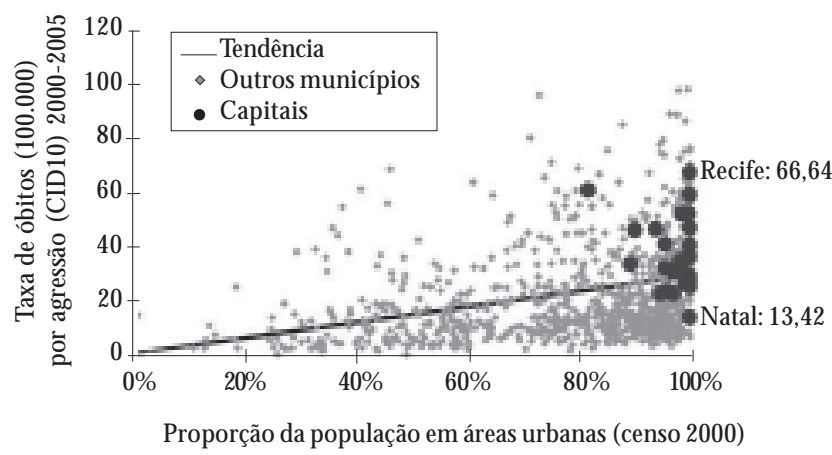

Fonte: DATASUS EIBGE.

Figura 2. Tendência linear entre a proporção da população vivendo em áreas urbanas de municípios brasileiros com mais de 30 mil habitantes e a taxa média de óbitos anual por agressão no período de 2000 a 2005, Brasil. gerir intervenções em nível municipal. Entretanto, ao considerar toda a cidade como unidade analítica de estudo, assume-se que os comportamentos ou características são homogêneos em toda a cidade, limitando a compreensão de "como" a cidade afeta a saúde de seus residentes, impossibilitando inferências para os indivíduos ${ }^{46}$.

Um quarto grupo de estudos comparativos tem apresentado técnicas epidemiológicas que muito contribuem para o entendimento de "como" o viver na cidade pode afetar a saúde. $M$ ais comumente, estes estudos focal izam a distribuição espacial de grupos de indivíduos, tipicamente descritos como pertencentes a uma vizinhança comum, seja ela de caráter administrativo-geográfico ou social. Denominados intra-urbanos ou entre vizinhanças, tais estudos permitem acessar informações correlacionadas com características da unidade espacial que podem estar associadas com eventos em saúde. Embora ainda com limitações, principalmente para os delineamentos ecológicos etransversais, as comparações intra-urbanas permitem ir além das metodologias anteriores. Exemplificam os estudos urbanos brasilei ros focados nos diferenciais intra-urbanos de eventos em saúde ligados à transmissão vetorial (dengue, leishmaniose), relacionados a comportamentos (homicídios egravidez em adolescentes) e a eventos evitáveis em saúdeinfantil (hospitalização em menores decinco anos por asma) $)^{28,56-59}$.

Investigando as diferenças intra-urbanas na mortalidade proporcional por grupos etários, apesar de comprovada redução das causas evitáveis de óbito em menores de um ano, no período de 2000 a 2005, observa-se que o declínio não é homogêneo quando sobreposto a um indicador composto de vulnerabilidade à saúde (IVS) nas diversas regiões da cidade. Áreas de muito elevado risco, coincidentes com regiões de extrema pobreza na cidade, apresentam diferenças importantes na mortalidade proporcional, principalmente na faixa equivalente a jovens adultos ( $\mathrm{Fi}$ guras $3 a$ e 3b), sugerindo queénasáreas de maior vulnerabilidadequeesta faixa etária contribui proporcionalmente com o maior número de óbitos. Evidentemente tal delineamento não permite inferências em nível de indivíduos mas outras estratégias, como aanálisemultinível, potencialmente permitirão considerar as associações incorporando variáveis de confusão tanto no nível de grupo quanto do indivíduo.

Embora tais comparações contribuam enormemente para o conhecimento das condições urbanas e suas implicações na saúde, estas pos- 
suem limitações quanto à generalização para outras cidades ou mesmo outras áreas urbanas da mesma cidade ${ }^{42}$.

\section{Consideraçõesfinais}

Perspectivas, oportunidades e desafios

A preocupação com a saúde nos grandes centros urbanos tem sido foco de atenção crescente nosúltimos anos, com reflexo positivo nas publicações científicas. No PubM ed, um dos maiscompletos bancos de dados de pesquisa bibliográfica,

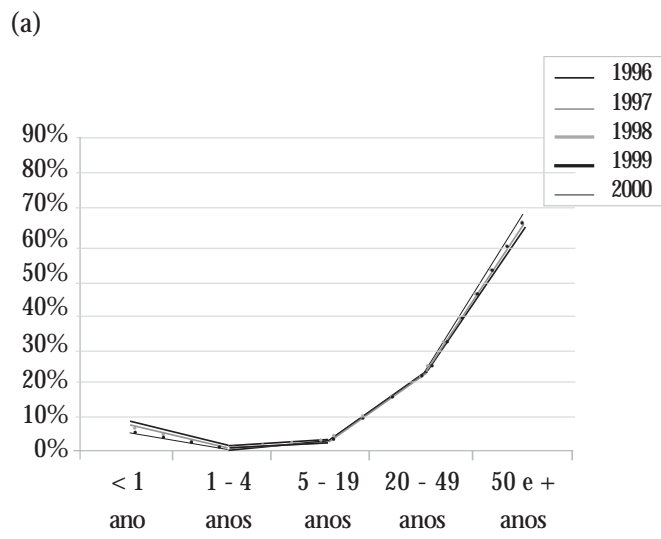

(b)

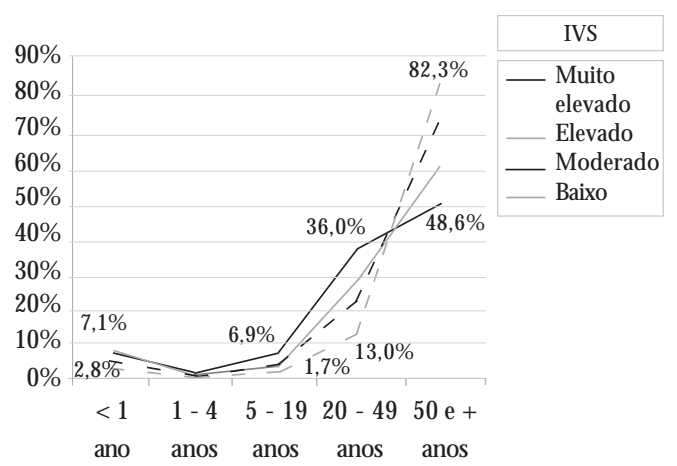

Fonte: SIM/SVS/M S-GEEPI/GVSI/SM SA/PBH .

Figura 3. (a) M ortalidade proporcional segundo grupos populacionais selecionados. Belo Horizonte, 1996 - 2000 e (b) estratificada pelo Índice de Vulnerabilidade à Saúde (IVS) do setor censitário de residência. Belo Horizonte, 2000. com cerca de 3.800 revistas científicas na área médica, foi possível verificar esta tendência. Utilizando o unitermo "urban health" e limitando a busca de artigos envolvendo pesquisas em seres humanos, observou-se um aumento significativo de publicações na última década. As oito primeiras publicações foram observadas na década de sessenta, passando para 432 nos anos setenta, 1.282 na década de oitenta, 4.995 na década de noventae 6.031 publicações nos últimos oito anos (janeiro de2000 a abril de 2008), correspondendo a taxas de publicação (por 10.000 artigos) de 0,15, $3,01,5,96,16,68$ e 18,90, respectivamente (Figura 4). Em 2008, o periódico Science dedicou todo um suplemento especial ao tema das cidades, explorando as ramificações das transformações urbanas, examinando como a cidades se conformam, os impactos da urbanização no ambiente, no crescimento econônico edemográfico ena saúde ${ }^{17,60,61}$. Também a questão cidades, urbanização e saúde foi tema de recenteeditorial do International Journal of Epidemiology ${ }^{62}$.

A mesma tendência pode ser observada com relação a artigos publicados no Brasil. No entanto, a produção científica ainda é muito escassa, ressaltando a importância de um enfoque mais direcionado a estetema. 0 primeiro artigo,

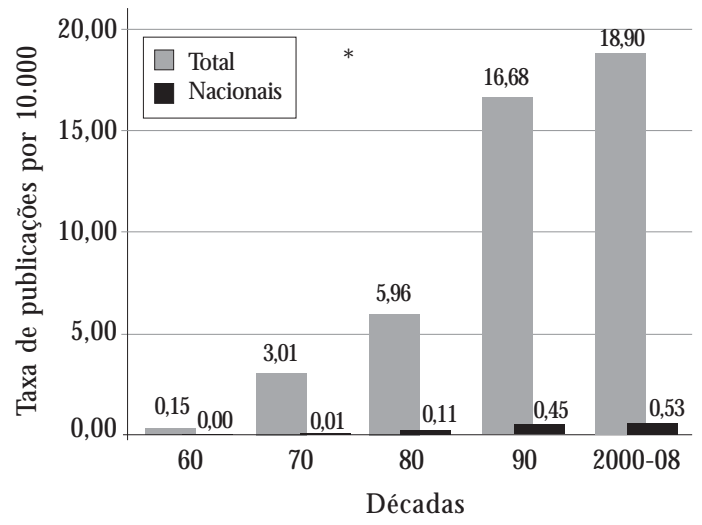

"Palavra chave: "urban health" (total); "urban health" e Brasil (nacionais).

Fonte: PubM ed (acessado 2008 M ai 05).

Figura 4. Distribuição temporal das taxas de publicação (por 10.000 artigos) contendo o unitermo "urban health", PubM ed, maio 2008. 
com dados brasileiros, foi publicado em 1979 , coincidindo com o grande crescimento urbano do país. Na década de oitenta, foram encontradas 24 publicações, passando para 132 nos anos noventa e 168 ao longo de janeiro de 2000 a abril de 2008, correspondendo a taxas de publicação (por 10.000 artigos) de 0,01, 0,11, 0,45 e 0,53, respectivamente (Figura 4 ).

Ao aumento significativo das publicações em saúde urbana observa-se, recentemente, uma mudança da ênfase inicial às doenças transmissíveis para abordagens, cada vez mais freqüentes, dos modos e estilos de vidas e con dições ambientais, tanto no mundo como no Brasil. Dentre os estudos brasileiros, o primeiro, publicado em 1979, abordou a temática das doenças infectoparasitárias. $\mathrm{N}$ a década seguinte, esta área temática correspondia a $38,0 \%$ das publicações, re duzindo-se para $29,0 \%$ no período de $2000-2008$.

São muitos os desafios a serem enfrentados. 0 estudo da saúde das populações no ambiente urbano resulta em uma nova perspectiva deabordagem da própria saúde pública requerendo transdisciplinaridade, em que pesquisadores combinem conhecimentos das suas várias áreas para desenvolver teorias, conceitos e métodos apropriados e direcionados para o objeto de estudo, a saúde urbana.

A decomposição do complexo conjunto de construtos que conformam o campo traz o desafio da adequação das definições básicas, e da validação das mesmas, para fins de pesquisa e prática da saúde pública. Um objetivo a ser perseguido refere-se à construção e desenvolvimento de ferramentas metodológicas de aferição que reflitam os vários construtos embutidos na saúde urbana. Estes construtos devem apresentar sensibilidade eespecificidade suficientes para detectarem diferenciais intra-urbanos passíveis de mensuração e suscetíveis à avaliação, capturando aspectos relevantes das políticas públicas (governabilidade) e desempenho social.

Repensar modelos de avaliações à saúde a partir de intervenções não dirigidas à saúde, desenvolver metodologias deanálises factíveiseacessíveis dentro de uma visão integradora, multidisciplinar e multisetorial, articulando planejamento, governança e saúde pública, são desafios relevantes. Os mesmos devem induzir maior investimento do corpo promotor da saúde pública quanto ànecessidade deresponder "como" e"para onde" vamos, em busca da saúde nas cidades.

\section{Colaboradores}

WT Caiaffa e FA Proietti trabalharam na concepção teórica, elaboração eredação final do texto; FR Ferreira, AD Ferreira, CDL Oliveira e VP Camargos participaram da organização, revisão bibliográfica e discussão teórica do texto.

\section{Agradecimentos}

Este artigo foi desenvolvido a partir da apresentação feita na mesa-redonda "Temas contemporâneos em saúde pública", realizada no Seminário Internacional da Universidade de São Paulo, por ocasião da celebração dos noventa anos da Faculdade deSaúde Pública, no dia 07 de abril de 2008, em São Paulo (proferida por WT Caiaffa). Os autores gostariam de agradecer ao Dr. David Vlahov pelas importantes contribuições durante a confecção destee de outros materiais em saúde urbana. WT Caiaffa e FA Proietti são recipientes de bolsas de produtividade do CN Pq. 


\section{Referências}

1. Satterthwaite D. Will most people live in cities? BM I 2000; 321(7269):1143-1145.

2. Satterthwaite D. Coping with rapid urban growth. London: Royal Institution of Chartered Surveyors; 2002.

3. United Nations Department of Economic Social Affairs/Population Division. World Urbanization Prospects: The 2007 revision. New York: UN; 2008.

4. Galea S, Vlahov D. Urban Health: Evidence, challenges and directions. Annu Rev Public Health 2005; 26:341-365.

5. Lefèbvre H. A revolução urbana. Belo H orizonte: Ed UFM G; 1999.

6. United Nations Department of Economic and Socia Affairs/Population Division. World Urbanization Prospects: The 2003 Revision. New York: UN; 2004.

7. Instituto Brasileiro de Geografia e Estatística. Departamento de População e Indicadores Sociais. Tendências demográficas: uma análise dos resultados da sinopse preliminar do censo demográfico 2000. Rio de Janeiro: IBGE; 2001.

8. Mata D, Deichmann U, Henderson JV, Lall SV, Wang HG. U m exame dos padrões de crescimento das cidades brasileiras. [Texto para discussão 1155]. Brasília: Instituto de Pesquisa e Economia Aplicada; 2006.

9. Prata PR. The Epidemiologic Transition in Brazil. Cad Saúde Pública 1992; 8(2):168-175.

10. Monte-M ór RL. What is urban in the contemporary world? Cad Saúde Pública 2005; 21(3):942-948.

11. UN Habitat. The Challenge of Slums-Global Report on H uman Settlements 2003. London: Earthscan; 2003. United Nations United Nations Department of Eco-

12. nomic Social Affairs/Population Division. World Urbanization Prospects: The 2005 revision. New York: UN; 2005.

13. United States Agency for International Development. Improving the health of the urban poor: learning from USAID experience. Washington: USAID; 2004.

14. Brito $F$, Souza J. Expansão urbana nas grandes metrópoles: o significado das migrações intrametropolitanas e da mobilidade pendular na reprodução da pobreza. São Paulo em Perspectiva 2005; 19(4):48-63.

15. Musso P. Télécommunications et philosophie des ré seaux. Paris: PUF; 1997.

16. M CM ichael AJ. The urban environment and health in a world of increasing globalization: issues for developing countries. Bull World Health Organ 2000; 78(9):1117-1126.

17. Baltimore D. Science for the globe. Science 2008 319(5864):697.

18. Omram AR. The epidemiologic transition: a theory of the epidemiology of population change. Milbank Memorial Fund Quarterly 1971; 49(Part 1):509-538.

19. Vlahov D, Galea S, Freudenberg N. Toward an urban health advantage. J Public Health $\mathrm{M}$ anag Pract 2005; 11(3):256-258.

20. Proietti FA, Caiaffa WT. Editorial Forum de Saúde U rbana: What is urban health? Cad Saúde Pública 2005; 21(3): 940-941.

21. Vlahov D, Galea S. Urban health: a new discipline. Lancet 2003; 362(9390):1091-1092.
22. Organização Pan-Americana da Saúde. Primer Foro Regional de Salud U rbana. Caminando hacia un marco conceptual de salud urbana y agenda para la acción en Ias Américas. La iniciativa de la OPS: Foro de Salud Pública de las Américas. Ciudad de M éxico, M éxico: 27-29 de noviembre, 2007.

23. Puffer RR, Griffith GW. Caracteristicas de la mortalidade urbana: informe de la investigación interamericana de mortalidade. Washington: OPAS; 1968.

24. World Health Organization. Center for Health Development Urbanization: A Global Health Challenge. Kobe, Japan: WHO Center for Health Development; 1996.

25. Sisco S. The 2nd International Conference on U rban $\mathrm{H}$ ealth in Review: building healthy cities for the 21st century. The Newsletter of the International Society for U rban H ealth 2004.

26. Ludermir AB. Editorial especial. Rev Bras Epidemio 2004; 7(1):8-10.

27. Vlahov D, Gálea S, Gibble E, Freudenberg N. Perspectives on urban conditions and population health. Cad Saúde Pública 2005; 21(3):949-957.

28. Caiaffa WT, Almeida M CM, Oliveira CL, Friche AAL, $M$ atos SG, Dias MAS, Cunha MCM, Pessanha E, Oliveira $C$. The urban environment from the health perspective: the case of Belo H orizonte, M inas Gerais, Brazil. Cad Saúde Pública 2005; 21(3):958-967.

29. World Health Organization. Our cities, our health, our future: acting on social determinants for health equity in urban settings. Report to the WHO Commission on Social Determinants of $\mathrm{H}$ ealth from the Knowledge Network on Urban Settings. Kobe, Japan: WHO; 2008.

30. Buss PM, Pellegrini Filho A. Iniquities in health in Brazil, our more serious illness: commentaries on the reference document and works of the Commission on Social Determinants of Health. Cad Saúde Pública 2006; 22(9):2005-2008.

31. Vlahov D, Quinn A, Putnam S, Proietti F, Caiaffa WT. Urban Health: Latin America and the Caribbean. Improving U rban Population Health Systems. Center for sustainable urban development. In: Bellagio Study and Conference Center. Innovations for an Urban World: a global urban summit. Bellagio, Italy: The Rockefeller Foundation; 2007. p.1-50

32. Vlahov D, Galea S. Urbanization, urbanicity, and health. J Urban Health 2002; 79(Suppl 1):S1-S12.

33. Wirth L. Urbanism as a way of life. Am J Sociol 1938; XLIV:1-24.

34. Instituto Brasileiro de Geografia e Estatística. Noções básicas de cartografia: elementos de representação - unidades político-administrativas [acessado 2008 Abr 02]. Disponível em: http://www.ibge.gov.br/ home/geociencias/cartografia/manual_nocoes/ elementos_representacao.html

35. Galea S, Schulrtz A. M ethodological considerations in the study of urban health: how do we best assess how cities affect health? In: Freudenberg N, Galea S, Vlahov D. Cities and the health of the public. Nashville: Vanderbilt University Press; 2006. p. 277-293.

36. Kjellstrom T, M ercado S, Sami M, Havemann K, I wao $S$. Achieving health equity in urban settings. J U rban Health 2007; 84(3 Suppl):i1-6. 
37. Dow K. Exploring differences in our common future(s): the meaning of vulnerability to global environmental change. Geoforum 1992; 23(3):417-436.

38. M arandola Jr E, Hogan DJ. Vulnerabilidades e riscos: entre geografia e demografia. R. Bras. Est. Pop. São Paulo 2005; 22(1):29-53.

39. Vlahov D, Freudenberg N, Proietti F, Ompad D Quinn A, Nandi V, Galea S. Urban as a determinant of health. J Urban Health 2007; 84(3 Suppl):i16-26.

40. Mindell J, Boltong A. M ini-symposium-public health observatories: Supporting health impact assessment in practice. Public Health 2005;119:246-252

41. Ompad DC, Galea S, Caiaffa WT, Vlahov D. Social determinants of the health of urban populations: methodologic considerations. J U rban H ealth 2007; 84(3 Suppl):i42-53.

42. Galea S, Vlahov D. Handbook of urban health: populations, methods, and practice. New York: Springer; 2005.

43. Freudenberg N, Galea S, Vlahov D. Cities and the health of the public. Nashville: Vanderbilt University Press; 2006.

44. Krieger N. Proximal, Distal, and the Politics of Causation: What's Level Got to Do With It? Public Health 2008; 98:221-230.

45. Pasquali L. Psicometria:teoria dos testes psicológicos Brasília: Prática; 2000.

46. Diez-Roux AV. Multilevel analysis in public health research. Annu Rev Public Health 2000; (21):171-192.

47. Diez-Roux AV. Invited commentary: places, people, and health. Am J Epidemiol 2002; 155(6):516-519.

48. Kawachi I, Berkamn LF. Social ties and mental health. J U rban H ealth 2001; 78(3):458-467.

49. Suminski RR, Petosa RL, Stevens E. A method for observing physical activity on residential sidewalks and streets. J U rban Health 2006; 83(3):434-443.

50. Raudenbush SW, Sampson RJ. Ecometrics: Toward a Science of Assessing Ecological Settings, With Application to the Systematic Social Observation of Neighborhoods. Sociol M ethodol 1999; 29(1):1-41.

51. Proietti FA, Oliveira CD, Ferreira FR, Ferreira AD Caiaffa WT. Unidade de contexto e observação social sistemática em saúde: Revendo conceitos e métodos. [No prelo].
52. Gershon RR, N eitzel R, Barrera M A, Akram M. Pilot survey of subway and bus stop noise levels. J U rban Health 2006; 83(5):802-812.

53. Gershon RR. Public transportation: advantages and challenges. J U rban Health 2005; 82(1):7-9.

54. Gouveia N, de Freitas CU, Martins LC, Marcilio IO. Respiratory and cardiovascular hospitalizations associated with air pollution in the city of São Paulo, Brazil. Cad Saúde Pública 2006; 22(12):2669-2677.

55. Novaes HMD, Braga PE, Schout D. Fatores associados à realização de exames preventivos para câncer nas mulheres brasileiras, PNAD 2003. Cienc Saúde Colet 2006; 11(4):1023-1035.

56. Oliveira CL, Assunção RM, Proietti FA, Reis IA. Spatial distribution of human and canine visceral leishmaniasis in Belo Horizonte, 1994-1997. Cad Saúde Pública 2001; 17(5):1231-1239.

57. Friche AAL, Caiaffa WT, César CC, Goulart LM F, Almeida MCM . Indicadores de saúde materno-infantil em Belo H orizonte, M inas Gerais, Brasil, 2001: análise dos diferenciais intra-urbanos. Cad Saúde Pública 2006; 22(9):1955-1965.

58. Almeida M CM, Caiaffa WT, Assunção RM, Proietti FA. Spatial Vulnerability to Dengue in a Brazilian Urban Area During a 7- Year Surveillance. J Urban Health 2007; 84(3):334-345.

59. Almeida M CM, Caiaffa WT, Assunção RM, Proietti FA. Dinâmica intra-urbana das epidemias de Dengue em Belo Horizonte, M G, 1996-2002. Cad Saúde Pública. [No prelo].

60. M ontgomery MR. The Urban Transformation of the Developing World. Science 2008; 319(5864): 761-764.

61. Dye C. Health and U rban Living. Science 2008; 319(5864):766-769.

62. Leon DA. Cities, urbanization and health. Int J Epidemiol 2008; 37(1):4-8.

Artigo apresentado em 05/05/2008

A provado em 13/05/2008

Versão final apresentada em 11/07/2008 